\title{
THE MOST DISTANT RADIO GALAXIES
}

\author{
K. C. Chambers \\ Sterrewacht, Postbus 9513, 2300RA, Leiden, The Netherlands
}

\begin{abstract}
High redshift radio galaxies $(z>1)$ are unique spatially extended probes of the galactic environment at the earliest observed epochs. Recent discoveries have unleashed a host of questions about their nature. Many new objects are being found, and their unusual properties are being uncovered. They generally exhibit the "alignment effect" wherein their highly elongated lumpy morphologies are lined up along the axes of their powerful radio sources. Evidence for both large star formation rates and scattered quasar light are found in the aligned continuum.
\end{abstract}

\section{ALIGNED RADIO GALAXIES}

The field of high redshift radio galaxies has undergone a revolution in the last three years. In the early eighties, high redshift $(1<z<2)$ radio galaxies were viewed as natural extentions of their low redshift counterparts - generally rather normal giant ellipticals. This of course made them attractive objects for studies of galaxy evolution and ultimately, it was hoped, for use in distinquishing between cosmological world models. With the near complete identification of the 3CR catalog by Spinrad /1/ and collaborators, it was possible to begin a number of detailed studies of the properties of thes objects. Lilly and Longair /2/ obtained infrared measurments of the 3CR galaxies, and this infrared data along with optical data obtained by the Berkely group was investigated with the newly developed Bruzual models /3/. The limitations of these models (coeval galaxy formation, oversimplified treatment of AGB stars, simple assumptions for unknowns such as the IMF, the star formation rate etc.) were appreciated but the ability of the models to fit the data gave hope that these objects were probing normal galaxy evolution.

However, in the late eighties a series of new discoveries and new ideas have forced us to recognize these objects as having extraordinary properties which are not understood. The first of these was the discovery by Chambers et al. /4/ and McCarthy et al. /5/ of the "alignment effect", the curious fact that at high redshift, (and only at high redshift) radio galaxies often have highly elongated optical continuum morphologies, and these optical extensions are reasonably well lined up with the axis of the powerful radio source.

At about the same time the long standing " $z=2$ " barrier for radio galaxies was broken with the discovery of $4 \mathrm{C} 40.36$ at $z=2.267$ by Chambers, Miley and van Breguel /6/ using a new technique of selecting candidates by their ultra steep radio spectrum. Lilly /7/ then made the discovery of the "1 Jy" source B2 $0902+34$ at $z=3.4$. At present the redshift record rests with one of the ultra steep spectrum sources, $4 \mathrm{C} 41.17$ at $z=3.8$, found by Chambers, Miley, and van Bruegel /8/. However, several groups are now pursing various samples and the number of known $z>1$ galaxies is rapidlly increasing.

With the discovery of $4 \mathrm{C} 41.17$ and $0902+34$ the infrared $\mathrm{K}$ band Hubble diagram has now been extended out to very high redshifts $/ 9 /$. However, the development and availability of infrared arrays led to the discovery by Chambers et al. /10/ and Eisenhardt and Chokshi 
/11/ that the alignment effect extends down into infrared (or well below the $4000 \AA$ break in the restframe). Thus for radio galaxies with $z>1$, some reasonable fraction of the light in the $\mathrm{K}$-band Hubble diagram is aligned with the radio source. This is difficult to understand if, as was previously thought, the $\mathrm{K}$ band light is dominated by an old stellar population.

However there may be other components, as indicated by the discovery of significant polarization in 3C368 /12,13/. A scattered component provides additional evidence for unified schemes of AGN's (e.g. Barthel /14/). In this picture the differences between radio loud quasars and radio galaxies are attributed entirely to their orientation with respect to the observer, i.e. a quasar is a radio galaxy seen within $\sim 20$ degrees of the radio axis. In radio galaxies the nucleus is assumed to be obscured along our line of sight but is beamed anisotropically in the plane of the sky and then scattered into our line of sight by some scattering medium associated with the host galaxy. The scattering could be due to dust, or perhaps more likely, electron scattering from a dense hot ICM /15/. However, Scarrot et al. /13/ point out that their imaging polarimetry observations of 3C368 indicate that beaming/scattering is insufficient to account for the alignment effect, and some additional source (starlight?) is required to account for all of the aligned continuum.

Aligned radio galaxies are not expected to contain much dust because of their large Lyman $\alpha$ surface brightnesses. Even a small amount of dust can extinguish UV resonance line photons such as Lyman $\alpha$. Nonetheless, dust may play an important role if it is clumped differently from the emission line gas. The $\mathrm{Ly} \alpha / \mathrm{H} \alpha$ ratio may be the best indicator of the presense of dust, but measurements of redshifted $\mathrm{H} \alpha$ will require the new generation of infrared spectrometers with very high sensitivity.

Aside from continuity arguments (unambigous radio galaxies are seen up to redshifts $z \sim 1$, they should have counterparts at higher redshifts) there are two pieces of (marginal) observational evidence that starlight makes up much of the light from high redshift radio galaxies. This evidence also suggests that (given a reasonable IMF cf. /16,17/) although the current star formation rate (SFR) is high, it must have been higher in the past.

First, Chambers and McCarthy /18/ found (modest) evidence for stellar absorption features in the rest frame ultraviolet spectra of high redshift radio galaxies. More significantly however, they found that among all the bright radio galaxies studied at high redshift, all have a significant turndown in the rest frame ultraviolet $\left(f_{\nu}\right)$ shortward of $1500 \AA$. For any reasonable initial mass function this cutoff suggests the star formation rate is decreasing or stopped otherwise the ultraviolet would be dominated by hotter stars /18/. (This sharp turndown is also hard to reconcile with a nonthermal origin for the UV).

Second, most high redshift radio galaxies have significant $4000 \AA$ breaks $/ 9,19 /$. This is a rough measure of the ratio of present to past star formation. If the galaxy were dominated by the current star formation, (i.e. the hypothetical primeval galaxy) the SED would be roughly flat in $f_{\nu}$, with little or no break at $4000 \AA$. Since they do have a break, then there must have been a time in the past when the star formation rate was higher.

The crucial question is when in past was the star formation higher? Lilly /7/ has suggested that the bulk of the star formation in $0902+34$ was at a much earlier epoch, and unrelated to the current burst. Chambers and Charlot /18/ pointed out that a decreasing SFR, such as required to fit the ultraviolet data $/ 18 /$, could also have produced a sufficient $4000 \AA$ break within the timescale of the current burst. This is consistent with the idea that the alignment effect is due (at least in part) to star formation triggered by the radio source.

Of course the presence of an underlying older giant elliptical can not be excluded given the large luminosities of these objects and the uncertainties of the SFR the IMF assumed in the models. But an older population is not required to obtain the observed magnitudes of the $4000 \AA$ breaks. Note that there is not much time available in popular cosmologies for these objects to have had an earlier star formation epoch which could produce an older population. For $H_{0}=50 \mathrm{~km} / \mathrm{sec} / \mathrm{Mpc}$ and $q_{0}=0.5$, the universe is only $1.25 \mathrm{Gyr}$ old at the redshift of $4 \mathrm{C} 41.17(z=3.8)$. If one believes in inflation, and therefore prefers $\Omega=1$, then any earlier, unrelated star forming epoch must have been at very high redshifts, $z>30$, and occured relatively rapidly anyway. 
An additonal constraint on the relative ages comes from the infrared alignments. Most of the aligned radio galaxies are also aligned in the infrared /10,11/. If the infrared light is dominated by an older stellar population, then at best there should be a slight 'anti' alignment (i.e. lined up with the minor axis) as there is at low redshift $/ 20,21 /$. The younger stellar population models, where a decreasing SFR produces a significant $4000 \AA$ break in short timescale, provide a plausible solution.

However there are some high redshift radio galaxies which are quite compact and have a rounder morphology the infrared. Lilly / $7 /$ has suggested that these objects are examples of an underlying older giant elliptical. Unfortunately, this is not necessarily a clean test of the radio source induced star formation scenario. In that picture one might expect such differences. If we are seeing the galaxy at the tail end of its formation process, then the inner regions of the galaxy were affected first as the radio source expands out into the protogalactic medium. The the outer regions are affected last, and are thus the bluest. Thus, if the star formation is occuring on a relatively short timescale, expanding in a deflagration from the center of the galaxy outwards with the radio source, one might expect the center to be older and redder than the edges. This suggests that, depending on the time which we happen to observe them, some objects could be more aligned in the blue than in the red.

Various scenarios have been proposed for how a radio source could trigger star formation. Rees /22/ and Begelman and Cioffi /23/ have proposed that the cocoons of "waste energy" inflated by the radio source engulfs and compressess cold gas clouds in a ripe protogalaxy, driving them over the Jeans limit and thus triggering star formation. De Young / 24/ has emphasized the role of entrainment by the jet, and Daly /25/ has discussed an analytical model of the shock processes. Chambers /26/ has emphasized that if the radio age is as long as $\sim 10^{8}$, then the amount of mass in the star burst can approach the mass of a giant elliptical. Rapid star formation can also have an effect on the subsequent dynamics of the galaxy. Since the radio cocoon can synchronize a star burst through out a protogalaxy on a timescale much faster than a free fall time, it can lead to ideal conditions for dissipationless collapse. In this way, radio source induced star formation can provide an excellent mechanism for establishing the inital conditions required to form spheroids $/ 26 /$.

One fundamental aspect of these considerations is the role that AGN's may have a played in the early stages of galaxy formation. Either thru the anisotropic ionizing continum, or the powerful radio source, or both, an AGN can drastically affect the state, and thus the cooling and collapse, of gas on galactic scales.

\section{VERY HIGH REDSHIFT RADIO GALAXIES}

In order to further investigate the galactic environment at high redshift, new and larger samples of high redshift radio galaxies are needed.

Previous work on distant radio galaxies has centered on various complete samples from flux-limited surveys. However, given the very large number of radio sources which become avaliable at fainter and fainter flux limits, it is desirable to develop techniques for pre-selecting the best candidates from such surveys. Our ultra steep radio spectrum method is based on the observed correlation between the radio spectra and luminosities of radio sources. Many authors have discussed the evidence for such a correlation $/ 27,28,29,30 /$, but perhaps the most suggestive came from an investigation of a sample of $4 \mathrm{C}$ radio sources having ultra-steep spectra /31/. Blumenthal and Miley /32/ found that the fraction of radio sources which had counterparts on the Palomar Sky Survey was a strong function of the radio spectral index, with almost no identifications for the steepest spectrum sources.

Based on this, we have begun an extensive radio, optical, and infrared investigation of these sources $/ 4,25 /$. Our ultra steep spectrum sample comprises $4 \mathrm{C}$ sources known to have spectral indices of $\alpha<-1$, between 178 and $5000 \mathrm{MHz}$, where $S_{\nu}=k \nu^{\alpha}$. The current status of our $4 \mathrm{C}$ ultra-steep spectrum survey is encouraging. Out of $334 \mathrm{C}$ ultra-steep spectrum sources, we have obtained 31 optical identifications, attempted optical spectroscopy of 32 , have found 16 with strong emission lines and with $z>0.5,8$ of these with $z>2$. 
We are also currently extending our ultra-steep spectrum survey to fainter flux levels using sources from the Parkes, Texas, Molonglo, 6C, and $8 \mathrm{C}$ surveys /33/. Preliminary results on a subsample of 50 objects indicate 26 of these are aligned, 14 are unresolved, 5 appear to be intermediate redshift rich clusters, 2 are weak ID's, and 3 are blank fields. Our first spectroscopic run indicates that at least 3 of the aligned objects are $z>2$.

Other groups pursing new high redshift radio galaxies are working from samples from a variety of surveys, including: the Parkes selected areas, The MIT-Greenbank survey, The Molonglo survey, the B3 survey, and miscellaneous other samples $/ 34,35 /$.

At the moment, there are presently known about 65 radio galaxies with $1<z<2,21$ with $2<z<3$ and 6 with $z>3$. discovered by the various groups, but the numbers are sure to increase rapidly in the next few years.

\section{SUMMARY}

Selecting radio sources for study on the basis of their ultra-steep radio spectrum has proven to be an extremely successful technique for locating very high redshift galaxies. This in turn has helped discover a remarkable property of high redshift radio galaxies, that their optical/infrared continua are generally aligned with their radio axes.

The extended optical and infrared continua of high redshift radio galaxies are probably dominated by starlight, although there also appears to be a scattered component from the nucleus.

Much work remains to be done, including addressing the question of how much of a role radio sources may have played in triggering star formation in massive galaxies.

\section{REFERENCES}

1. Spinrad, H. Djorgovski, S., Marr, J., Aguilar, L. 1985a, Pub. A.S.P., 97, 932

2. Lilly, S. J., Longair, M. S. 1984, M.N.R.A.S., 211,833

3. Bruzual, G. 1983 Ap. J., 273, 105.

4. Chambers, K.C., Miley, G.K., and van Breugel, W. 1987 Nature, 329, 604.

5. McCarthy, P. J., Van Breugel, W., Spinrad, H., and Djorgovski, S. 1987, Ap. J. (Letters), 321, L29

6. Chambers, K.C., Miley, G.K., and van Breugel, W. 1988, Ap. J., 327, L47

7. Lilly, S. J. 1988, Ap. J., 333, 161.

8. Chambers, K.C., Miley, G.K., and van Breugel, W. 1990 Ap. J., 363, 27

9. Lilly, S. J. 1989, Ap. J., 340, 77.

10. Chambers, K.C., Miley, G.K., and Joyce, R. R. 1988b, Ap. J., 329, L75

11. Eisenhardt, P., Chokshi, A., 1990, Ap. J., 351, L9

12. di Serego Alighieri, S., Fosbury, R, Tadhunter, C., 1989, Nature, in press

13. Scarrot, S. M., Rolph, C. D., and Tadhunter, C. N., 1990 M.N.R.A.S., 243, 5p

14. Barthel, P.D., 1989, Ap. J., 336, 606

15. Fabian, A.C., 1989, M.N.R.A.S., 238, 41p

16. Bithell, M., and Rees, M., 1989 preprint

17. Chambers, K.C., and Miley, G. K., Evolution of the Universe of Galaxies, Edwin Hubble Centential Symposium, ed. R. Kron, Astro. Society of the Pacific Conference Series, p. 373

18. Chambers, K.C., and McCarthy, P.J., 1990, Ap. J. (Letters), 354, L9

19. Chambers, K.C., and Charlot, S., 1990, Ap. J. (Letters), 348, L1

20. Palimaka, J.J., Bridle, A.H., Fomalont, E. B., Brandie, G. W., 1979, Ap. J., 231, L7

21. Guthrie, B. N. G., 1979, M.N.R.A.S., 187, 581

22. Rees, M.J. 1989, M.N.R.A.S., 239, 1p.

23. Begelman, M.C., and Cioffi, D.F., 1989 Ap. J. (Letters), 345, L21

24. DeYoung, D., 1989 Ap. J. (Letters), 342, L59

25. Daly, R., 1990, Ap. J., 355, 416 
26. Chambers, K.C., 1989, Ph.D. Thesis, The Johns Hopkins University

27. Heeschen, D., 1960, Pub. A.S.P., 72, 368

28. Veron, M.P., Beron, P., Witzel, A., 1972, Astr. Ap., 18, 82

29. Macleod, J.M., Doherty, L.H., 1972, Nature , 238, 88

30. Bridle, A.H., Kesteven, M.J.L. Guindon, B., 1972, Ap. J. (Letters), 11,27

31. Tielens, S., Miley, G., Willis, A., 1979, Astr. Ap. Suppl., 35,153

32. Blumenthal, G., Miley, G.K., 1979, Astr. Ap., 80, 13

33. Miley, G.K, et al. 1989, ESO Messenger 56, 16

34. Spinrad, H., private communication

35. McCarthy, P.J., Kapahi, V. K., van Breugel, W., Subrahmanya, C. R., 1990, preprint 